\title{
Geochemically-Assisted Fracture: A phase-field study
}

\author{
PANIA NEWELL ${ }^{1}$, LOUIS SCHULER ${ }^{2}$ AND ANASTASIA \\ G. ILGEN ${ }^{3}$
}

${ }^{1}$ The University of Utah

${ }^{2}$ Ecole Normale Supérieure Paris-Saclay

${ }^{3}$ Sandia National Laboratories

Presenting Author: Pania.Newell@utah.edu

Geochemically-driven rock deformation may arise due to rock alteration via hydration, oxidation, carbonation, and dissolution/precipitation reactions under confining pressures. Such alterations lead to irreversible changes in mechanical properties and potentially changes in volume that leads to fracture propagation over time. Chemical dissolution changes the fracture properties and affects the mechanical strength of fractures. Due to the strong coupling between mechanics and chemistry, it is often challenging to determine how dissolution impacts fracture initiation and propagation. This presentation focuses on chemically-driven fractures and shows how the stateof-the-art phase-field fracture model can be used to capture such coupled processes in porous systems. We introduce a chemical damage parameter which is coupled with the mechanical damage parameter obtained from the phase-field equation. The chemical damage parameter is also coupled with the change in porosity due to dissolution occurring in the porous material. We will demonstrate how this coupled formulation enables us to gain an understanding of the impact of calcite dissolution on fracture behavior in carbonate rocks. We also discuss how this chemical dissolution impacts crack speed and directionality.

Sandia National Laboratories is a multimission laboratory managed and operated by National Technology and Engineering Solutions of Sandia, LLC., a wholly owned subsidiary of Honeywell International, Inc., for the U.S. Department of Energy's National Nuclear Security Administration under contract DE-NA-0003525. Disclaimer: This presentation describes objective technical results and analysis. Any subjective views or opinions that might be expressed in the presentation do not necessarily represent the views of the U.S. Department of Energy or the United States Government.

\section{Reference:}

Schuler, A. G. Ilgen, P. Newell, Chemo-mechanical phasefield modeling of dissolution-assisted fracture, Computer Methods in Applied Mechanics and Engineering, V. 362, 2020, 112838 . 